\title{
LINEARITY DEFECT AND REGULARITY OVER A KOSZUL ALGEBRA
}

\author{
KOHJI YANAGAWA*
}

\begin{abstract}
Let $A=\bigoplus_{i \in \mathrm{N}} A_{i}$ be a Koszul algebra over a field $K=A_{0}$, and ${ }^{*} \bmod A$ the category of finitely generated graded left $A$-modules. The linearity defect $\operatorname{ld}_{A}(M)$ of $M \in{ }^{*} \bmod A$ is an invariant defined by Herzog and Iyengar. An exterior algebra $E$ is a Koszul algebra which is the Koszul dual of a polynomial ring. Eisenbud et al. showed that $\operatorname{ld}_{E}(M)<\infty$ for all $M \in{ }^{*} \bmod E$. Improving this, we show that the Koszul dual $A^{!}$of a Koszul commutative algebra $A$ satisfies the following.

- Let $M \in{ }^{*} \bmod A^{!}$. If $\left\{\operatorname{dim}_{K} M_{i} \mid i \in \mathrm{Z}\right\}$ is bounded, then $\operatorname{ld}_{A^{!}}(M)<\infty$.

- If $A$ is complete intersection, then $\operatorname{reg}_{A^{!}}(M)<\infty$ and $\operatorname{ld}_{A^{!}}(M)<\infty$ for all $M \in{ }^{*} \bmod A^{!}$.

- If $E=\bigwedge\left\langle y_{1}, \ldots, y_{n}\right\rangle$ is an exterior algebra, then $\operatorname{ld}_{E}(M) \leq c^{n !} 2^{(n-1) !}$ for $M \in{ }^{*} \bmod E$ with $c:=\max \left\{\operatorname{dim}_{K} M_{i} \mid i \in \mathrm{Z}\right\}$.
\end{abstract}

\section{Introduction}

Let $A=\bigoplus_{i \in \mathrm{N}} A_{i}$ be a (not necessarily commutative) graded algebra over a field $K:=A_{0}$ with $\operatorname{dim}_{K} A_{i}<\infty$ for all $i \in \mathrm{N}$, and *mod $A$ the category of finitely generated graded left $A$-modules. Throughout this paper, we assume that $A$ is $K o s z u l$, that is, $K=A / \bigoplus_{i \geq 1} A_{i}$ has a graded free resolution of the form

$\cdots \longrightarrow A(-i)^{\beta_{i}(K)} \longrightarrow \cdots \longrightarrow A(-2)^{\beta_{2}(K)}$

$$
\longrightarrow A(-1)^{\beta_{1}(K)} \longrightarrow A \longrightarrow K \longrightarrow 0 .
$$

Koszul duality is a certain derived equivalence between $A$ and its Koszul dual algebra $A^{!}:=\operatorname{Ext}_{A}^{\bullet}(K, K)$.

For $M \in{ }^{*} \bmod A$, we have its minimal graded free resolution $\cdots \rightarrow P_{1} \rightarrow$ $P_{0} \rightarrow M \rightarrow 0$, and natural numbers $\beta_{i, j}(M)$ such that $P_{i} \cong \bigoplus_{j \in Z} A(-j)^{\beta_{i, j}(M)}$.

We call

$$
\operatorname{reg}_{A}(M):=\sup \left\{j-i \mid i \in \mathrm{N}, j \in \mathrm{Z} \text { with } \beta_{i, j}(M) \neq 0\right\}
$$

\footnotetext{
* The author is partially supported by Grant-in-Aid for Scientific Research (c) (no.19540028).
} Received November 14, 2007. 
the regularity of $M$. If $A$ is not left noetherian, then there is some $M \in{ }^{*} \bmod A$ such that $\sum_{j \in \mathrm{Z}} \beta_{1, j}(M)=\infty$. In this case, $\operatorname{reg}_{A}(M)=\infty$.

When $A$ is a polynomial ring, $\operatorname{reg}_{A}(M)$ is called the Castelnuovo-Mumford regularity of $M$, and has been deeply studied from both geometric and computational interest. Even for a general Koszul algebra $A, \operatorname{reg}_{A}(M)$ is still an interesting invariant closely related to Koszul duality (see Theorem 3.3 below).

Let $P_{\bullet}$ be a minimal graded free resolution of $M \in{ }^{*} \bmod A$. The linear $\operatorname{part} \operatorname{lin}\left(P_{\bullet}\right)$ of $P_{\bullet}$ is the chain complex such that $\operatorname{lin}\left(P_{\bullet}\right)_{i}=P_{i}$ for all $i$ and its differential maps are given by erasing all the entries of degree $\geq 2$ from the matrices representing the differentials of $P_{\boldsymbol{0}}$. According to Herzog-Iyengar [11], we call

$$
\operatorname{ld}_{A}(M):=\sup \left\{i \mid H_{i}\left(\operatorname{lin}\left(P_{\bullet}\right)\right) \neq 0\right\}
$$

the linearity defect of $M$. This invariant is related to the regularity via Koszul duality (see Theorem 3.9 below).

In $\S 4$, we mainly treat a Koszul commutative algebra $A$ and its dual $A$ ! Even in this case, it can occur that $\operatorname{ld}_{A}(M)=\infty$ for some $M \in{ }^{*} \bmod A$ ([11]), while Avramov-Eisenbud [1] showed that $\operatorname{reg}_{A}(M)<\infty$ for all $M \in{ }^{*} \bmod A$. On the other hand, Herzog-Iyengar [11] proved that if $A$ is complete intersection or Golod then $\operatorname{ld}_{A}(M)<\infty$ for all $M \in{ }^{*} \bmod A$. Initiated by these results, we will show the following.

Theorem A. Let A be a Koszul commutative algebra (more generally, a Koszul algebra with $\operatorname{reg}_{A}(M)<\infty$ for all $\left.M \in{ }^{*} \bmod A\right)$. Then we have;

(1) Let $N \in{ }^{*} \bmod A^{\text {! If }} \operatorname{reg}_{A^{!}}(N)<\infty$ (e.g., $\left.\operatorname{dim}_{K} N<\infty\right)$, then $\operatorname{ld}_{A^{!}}(N)<\infty$.

(2) The following conditions are equivalent.

(a) $\operatorname{ld}_{A}(M)<\infty$ for all $M \in{ }^{*} \bmod A$.

(a') $\operatorname{ld}_{A}(M)<\infty$ for all $M \in{ }^{*} \bmod A$ with $M=\bigoplus_{i=0,1} M_{i}$.

(b) If $N \in{ }^{*} \bmod A^{!}$has a finite presentation, then $\operatorname{reg}_{A^{!}}(N)<\infty$.

In Theorem A (2), the implications $(a) \Rightarrow\left(a^{\prime}\right) \Leftrightarrow(b)$ hold for a general Koszul algebra.

When $A$ is commutative, Bøgvad and Halperin [4] showed that $A^{!}$is noetherian if and only if $A$ is complete intersection. Moreover, by Backelin and Roos [2, Corollary 2], if $A$ is a Koszul complete intersection then $\operatorname{reg}_{A^{!}}(N)<\infty$ for all $N \in{ }^{*} \bmod A !$. (Since $A^{!}$admits a balanced dualizing complex, we can explain this also by [12].) So, in this case, we have $\operatorname{ld}_{A}(M)<\infty$ for all $M \in{ }^{*} \bmod A$ by Theorem A (2). This is a part of the above result of Herzog and Iyengar. Their proof takes a slightly different approach, but is also based on a similar result in [2]. 
Let *fp $A$ ! be the full subcategory of * mod $A$ ! consisting of finitely presented modules.

Theorem B. If $A$ is a Koszul algebra such that $\operatorname{ld}_{A}(M)<\infty$ for all $M \in{ }^{*} \bmod A$, then $A^{!}$is left coherent (in the graded context), and ${ }^{*} \mathrm{fp} A^{!}$is an abelian category. If further $A$ is commutative, then Koszul duality gives

$$
\mathscr{D}^{b}\left({ }^{*} \bmod A\right) \cong \mathscr{D}^{b}\left({ }^{*} \mathrm{fp} A^{!}\right)^{\mathrm{op}} .
$$

Corollary C. Let A be a Koszul commutative algebra. If A is Golod, then we have $\mathscr{D}^{b}\left({ }^{*} \bmod A\right) \cong \mathscr{D}^{b}\left({ }^{*} \mathrm{fp} A^{!}\right)^{\text {op }}$. If $A$ is a complete intersection, then we have $\mathscr{D}^{b}\left({ }^{*} \bmod A\right) \cong \mathscr{D}^{b}\left({ }^{*} \bmod A^{!}\right)^{\text {op }}$.

Let $E:=\bigwedge\left\langle y_{1}, \ldots, y_{n}\right\rangle$ be an exterior algebra. Eisenbud et al. [7] showed that $\operatorname{ld}_{E}(N)<\infty$ for all $N \in{ }^{*} \bmod E$ (now this is a special case of Theorem A, since $E$ is the Koszul dual of a polynomial ring $\left.S:=K\left[x_{1}, \ldots, x_{n}\right]\right)$. If $n \geq 2$, then $\sup \left\{\operatorname{ld}_{E}(N) \mid N \in{ }^{*} \bmod E\right\}=\infty$. On the other hand, we will see that

$$
\operatorname{ld}_{E}(N) \leq c^{n !} 2^{(n-1) !} \quad\left(c:=\max \left\{\operatorname{dim}_{K} N_{i} \mid i \in \mathrm{Z}\right\}\right)
$$

for $N \in{ }^{*} \bmod E$.

To prove this, we use (a special case of) a result of Brodmann and Lashgari ([6, Theorem 2.6]) stating that if a submodule $M \subset S^{\oplus c}$ is generated by elements of degree 1 then $\operatorname{reg}_{S}(M) \leq c^{n !} 2^{(n-1) !}$. But a computer experiment suggests that the bound (1) could be very far from sharp. For example, if $I \subset E$ is a monomial ideal then we have $\operatorname{ld}_{E}(E / I) \leq \max \{n-2,1\}$ ([15]). This does not hold for general graded ideals. We have a graded ideal $I \subset E$ with $n=6$ and $\operatorname{ld}_{E}(E / I)=9$. It is not hard to find similar examples, but these are still much lower than the value given in (1).

\section{Koszul Algebras and Koszul Duality}

Let $A=\bigoplus_{i \in \mathrm{N}} A_{i}$ be a graded algebra over a field $K:=A_{0}$ with $\operatorname{dim}_{K} A_{i}<\infty$ for all $i \in \mathrm{N},{ }^{*}$ Mod $A$ the category of graded left $A$-modules, and ${ }^{*} \bmod A$ the full subcategory of * $\operatorname{Mod} A$ consisting of finitely generated modules. We say $M=\bigoplus_{i \in Z} M_{i} \in{ }^{*} \operatorname{Mod} A$ is quasi-finite, if $\operatorname{dim}_{K} M_{i}<\infty$ for all $i$ and $M_{i}=0$ for $i \ll 0$. If $M \in{ }^{*} \bmod A$, then it is clearly quasi-finite. We denote the full subcategory of ${ }^{*} \operatorname{Mod} A$ consisting of quasi-finite modules by qf $A$. Clearly, of $A$ is an abelian category with enough projectives. For $M \in{ }^{*} \operatorname{Mod} A$ and $j \in \mathrm{Z}, M(j)$ denotes the shifted module of $M$ with $M(j)_{i}=M_{i+j}$. For $M, N \in{ }^{*} \operatorname{Mod} A$, set $\underline{\operatorname{Hom}}_{A}(M, N):=\bigoplus_{i \in Z} \operatorname{Hom}^{*} \operatorname{Mod} A(M, N(i))$ to be a graded $K$-vector space with $\underline{\operatorname{Hom}}_{A}(M, N)_{i}=\operatorname{Hom}_{*}{ }_{\operatorname{Mod} A}(M, N(i))$. Similarly, we also define $\underline{\operatorname{Ext}}_{A}^{j}(M, N)$. 
Let $\mathscr{C}$ (qf $A$ ) be the homotopy category of cochain complexes in qf $A$, and $\mathscr{C}^{-}(\mathrm{qf} A)$ its full subcategory consisting of complexes which are bounded above (i.e., $X^{\bullet} \in \mathscr{C}\left(\mathrm{qf} A\right.$ ) with $X^{i}=0$ for $i \gg 0$ ). We say $P^{\bullet} \in \mathscr{C}^{-}$(qf $A$ ) is a free resolution of $X^{\bullet} \in \mathscr{C}^{-}$(qf $A$ ), if each $P^{i}$ is a free module and there is a quasi-isomorphism $P^{\bullet} \rightarrow X^{\bullet}$. We say a free resolution $P^{\bullet}$ is minimal, if $\partial\left(P^{i}\right) \subset \mathfrak{m} P^{i+1}$ for all $i$. Here $\partial$ denotes the differential map, and $\mathfrak{m}:=$ $\bigoplus_{i>0} A_{i}$ is the graded maximal ideal. Any $X^{\bullet} \in \mathscr{C}^{-}$(qf $A$ ) has a minimal free resolution, which is unique up to isomorphism of cochain complexes.

Regard $K=A / \mathfrak{m}$ as a graded left $A$-module, and set

$$
\beta_{j}^{i}\left(X^{\bullet}\right):=\operatorname{dim}_{K} \underline{\operatorname{Ext}}_{A}^{-i}\left(X^{\bullet}, K\right)_{-j} \quad \text { and } \quad \beta^{i}\left(X^{\bullet}\right):=\sum_{j \in Z} \beta_{j}^{i}\left(X^{\bullet}\right)
$$

for $X^{\bullet} \in \mathscr{C}^{-}$(qf $A$ ) and $i, j \in \mathrm{Z}$. If $P^{\bullet}$ is a minimal free resolution of $X^{\bullet}$, then $P^{i} \cong \bigoplus_{j \in \mathrm{Z}} A(-j)^{\beta_{j}^{i}\left(X^{\bullet}\right)}$ for each $i \in \mathrm{Z}$. Clearly, $\beta_{j}^{i}\left(X^{\bullet}\right)<\infty$ for all $i, j$.

Following the usual convention, we often describe (the invariants of) a free resolution of a module $M \in$ qf $A$ in the homological manner. So we have $\beta_{i, j}(M)=\beta_{j}^{-i}(M)$, and a minimal free resolution of $M$ is of the form

$$
P_{\bullet}: \cdots \longrightarrow \bigoplus_{j \in Z} A(-j)^{\beta_{1, j}(M)} \longrightarrow \bigoplus_{j \in Z} A(-j)^{\beta_{0, j}(M)} \longrightarrow M \longrightarrow 0 .
$$

We say $A$ is $K o s z u l$, if $\beta_{i, j}(K) \neq 0$ implies $i=j$, in other words, $K$ has a graded free resolution of the form

$\cdots \longrightarrow A(-i)^{\beta_{i}(K)} \longrightarrow \cdots \longrightarrow A(-2)^{\beta_{2}(K)}$

$$
\longrightarrow A(-1)^{\beta_{1}(K)} \longrightarrow A \longrightarrow K \longrightarrow 0 .
$$

Even if we regard $K$ as a right $A$-module, we get the equivalent definition.

The polynomial ring $K\left[x_{1}, \ldots, x_{n}\right]$ and the exterior algebra $\bigwedge\left\langle y_{1}, \ldots, y_{n}\right\rangle$ are primary examples of Koszul algebras. Of course, there are many other important examples. In the noncommutative case, some of them are not left (or right) noetherian. In the rest of the paper, we assume that $A$ is Koszul.

Koszul duality is a derived equivalence between a Koszul algebra $A$ and its dual $A$ !. A standard reference of this subject is Beilinson et al. [3]. But, in the present paper, we follow the convention of Mori [14].

Recall that Yoneda product makes $A^{!}:=\bigoplus_{i \in \mathrm{N}} \operatorname{Ext}_{A}^{i}(K, K)$ a graded $K$ algebra. (In the convention of [3], $A^{!}$denotes the opposite algebra of our $A^{!}$. So the reader should be careful.) If $A$ is Koszul, then so is $A^{!}$and we have $\left(A^{!}\right)^{!} \cong A$. The Koszul dual of the polynomial ring $S:=K\left[x_{1}, \ldots, x_{n}\right]$ is the exterior algebra $E:=\bigwedge\left\langle y_{1}, \ldots, y_{n}\right\rangle$. In this case, since $S$ is regular and noetherian, Koszul duality is very simple. It gives an equivalence 
$\mathscr{D}^{b}\left({ }^{*} \bmod S\right) \cong \mathscr{D}^{b}\left({ }^{*} \bmod E\right)$ of the bounded derived categories. This equivalence is sometimes called Bernstein-Gel'fand-Gel'fand correspondence ( $B G G$ correspondence for short). In the general case, the description of Koszul duality is slightly technical. For example, if $A$ is not left noetherian, then * mod $A$ is not an abelian category. So we have to treat qf $A$.

Let $\mathscr{C}^{\uparrow}$ (qf $A$ ) be the full subcategory of $\mathscr{C}(\mathrm{qf} A)$ (and $\mathscr{C}^{-}$(qf $\left.A\right)$ ) consisting of complexes $X^{\bullet}$ satisfying

$$
X_{j}^{i}=0 \quad \text { for } i \gg 0 \text { and for } i+j \ll 0 .
$$

And let $\mathscr{D}^{\uparrow}$ (qf $A$ ) be the localization of $\mathscr{C}^{\uparrow}$ (qf $A$ ) at quasi-isomorphisms. By the usual argument, we see that $\mathscr{D} \uparrow$ (qf $A$ ) is equivalent to the full subcategory of the derived category $\mathscr{D}(\mathrm{qf} A)$ (and $\mathscr{D}^{-}(\mathrm{qf} A)$ ) consisting of the complex $X^{\bullet}$ such that

$$
H^{i}\left(X^{\bullet}\right)_{j}=0 \quad \text { for } i \gg 0 \text { and for } i+j \ll 0 .
$$

We also see that $\mathscr{D}^{\uparrow}$ (qf $A$ ) is a triangulated subcategory of $\mathscr{D}(\mathrm{qf} A)$.

We write $V^{*}$ for the dual space of a $K$-vector space $V$. Note that if $M \in$ ${ }^{*}$ Mod $A$ then $M^{*}:=\bigoplus_{i \in \mathrm{Z}}\left(M_{i}\right)^{*}$ is a right $A$-module. And we fix a basis $\left\{x_{\lambda}\right\}$ of $A_{1}$ and its dual basis $\left\{y_{\lambda}\right\}$ of $\left(A_{1}\right)^{*}\left(=\left(A^{!}\right)_{1}\right)$. Let $\left(X^{\bullet}, \partial\right) \in \mathscr{C}^{\uparrow}(\mathrm{qf} A)$. In this notation, we define the contravariant functor $F_{A}: \mathscr{C}^{\uparrow}$ (qf $A$ ) $\rightarrow \mathscr{C}^{\uparrow}$ (qf $A^{!}$) as follows.

$$
F_{A}\left(X^{\bullet}\right)_{q}^{p}=\bigoplus A_{q+j}^{!} \otimes_{K}\left(X_{-j}^{j-p}\right)^{*}
$$

with the differential $d=d^{\prime}+d^{\prime \prime}$ given by

$$
\begin{aligned}
d^{\prime}: A_{q+j}^{!} \otimes_{K}\left(X_{-j}^{j-p}\right)^{*} & \ni a \otimes m \\
& \longmapsto(-1)^{p} \sum a y_{\lambda} \otimes m x_{\lambda} \in A_{q+j+1}^{!} \otimes_{K}\left(X_{-j-1}^{j-p}\right)^{*}
\end{aligned}
$$

and

$$
d^{\prime \prime}: A_{q+j}^{!} \otimes_{K}\left(X_{-j}^{j-p}\right)^{*} \ni a \otimes m \longmapsto a \otimes \partial^{*}(m) \in A_{q+j}^{!} \otimes_{K}\left(X_{-j}^{j-p-1}\right)^{*} .
$$

The contravariant functor $F_{A^{!}}: \mathscr{C}^{\uparrow}\left(\mathrm{qf} A^{!}\right) \rightarrow \mathscr{C}^{\uparrow}(\mathrm{qf} A)$ is given in a similar way. (More precisely, the construction is different, but the result is similar. See the remark below.) They induce the contravariant functors $\mathscr{F}_{A}: \mathscr{D}^{\uparrow}$ (qf $\left.A\right) \rightarrow$ $\mathscr{D}^{\uparrow}\left(\right.$ qf $\left.A^{!}\right)$and $\mathscr{F}_{A} !: \mathscr{D}^{\uparrow}\left(\right.$ qf $\left.A^{!}\right) \rightarrow \mathscr{D}^{\uparrow}($ qf $A$ ).

REMARK 2.1. In [14], two Koszul duality functors are defined individually. The functor denoted by $\bar{E}_{A}$ is the same as our $\mathscr{F}_{A}$. The other one which is denoted by $\tilde{E}_{A}$ is defined using the operations $\underline{\operatorname{Hom}}_{K}\left(A^{!},-\right)$and $\underline{\operatorname{Hom}}_{K}(-, K)$. 
But, in our case, it coincides with $F_{A}$ except the convention of the sign \pm 1 . So we do not give the precise definition of $\tilde{E}_{A}$ here.

Theorem 2.2 (Koszul duality. cf. [3], [14]). The contravariant functors $\mathscr{F}_{A}$ and $\mathscr{F}_{A}$ ! give an equivalence

$$
\mathscr{D}^{\uparrow}(\mathrm{qf} A) \cong \mathscr{D}^{\uparrow}\left(\mathrm{qf} A^{!}\right)^{\mathrm{op}} .
$$

The next result easily follows from Theorem 2.2 and the fact that $\mathscr{F}_{A}(K)=$ $A^{!}$.

Lemma 2.3 (cf. [14, Lemma 2.8]). For $X^{\bullet} \in \mathscr{D}^{\uparrow}(\mathrm{qf} A$ ), we have

$$
\beta_{j}^{i}\left(X^{\bullet}\right)=\operatorname{dim} H^{-i-j}\left(\mathscr{F}_{A}\left(X^{\bullet}\right)\right)_{j} .
$$

\section{Regularity and Linearity Defect}

Throughout this section, $A=\bigoplus_{i \in \mathrm{N}} A_{i}$ is a Koszul algebra.

Definition 3.1. For $X^{\bullet} \in \mathscr{D}^{\uparrow}$ (qf $A$ ), we call

$$
\operatorname{reg}_{A}\left(X^{\bullet}\right):=\sup \left\{i+j \mid i, j \in \mathrm{Z} \text { with } \beta_{j}^{i}\left(X^{\bullet}\right) \neq 0\right\}
$$

the regularity of $X^{\bullet}$. For convenience, we set the regularity of the 0 module to be $-\infty$.

If $M \in \mathrm{qf} A$ is not finitely generated, then $\beta_{0, j}(M) \neq 0$ for arbitrary large $j$ and $\operatorname{reg}_{A}(M)=\infty$.

If $A$ is a polynomial ring $K\left[x_{1}, \ldots, x_{n}\right]$ (more generally, $A$ is $A S$ regular $)$, then $\operatorname{reg}_{A}\left(X^{\bullet}\right)$ of $X^{\bullet} \in \mathscr{D}^{b}\left({ }^{*} \bmod A\right)$ can be defined in terms of the local cohomology modules $H_{\mathfrak{m}}^{i}\left(X^{\bullet}\right)$, see [8], [12], [19]. If $A$ is commutative, it is known that $\operatorname{reg}_{A}(M)<\infty$ for all $M \in{ }^{*} \bmod A$ (see Theorem 4.2 below). But this is not true in the non-commutative case. In fact, if $A$ is not left noetherian, then $A$ has a graded left ideal $I$ which is not finitely generated, that is, $\beta_{1}(A / I)=\beta_{0}(I)=\infty$. Hence, if $A$ is not left noetherian, then $\operatorname{reg}_{A}(M)=\infty$ for some $M \in{ }^{*} \bmod A$. The author does not know any example $M \in{ }^{*} \bmod A$ such that $\beta_{i}(M)<\infty$ for all $i$ but $\operatorname{reg}_{A}(M)=\infty$.

LEMMA 3.2 .

(1) For $M \in$ qf $A$, we have

$$
\operatorname{reg}_{A}(M)<\infty \Rightarrow \beta_{i}(M)<\infty \text { for all } i \Rightarrow M \text { has a finite presentation. }
$$

(2) If $X^{\bullet} \rightarrow Y^{\bullet} \rightarrow Z^{\bullet} \rightarrow X^{\bullet}[1]$ is a triangle in $\mathscr{D}^{\uparrow}$ (qf $A$ ), then we have

$$
\operatorname{reg}_{A}\left(Y^{\bullet}\right) \leq \max \left\{\operatorname{reg}_{A}\left(X^{\bullet}\right), \operatorname{reg}_{A}\left(Z^{\bullet}\right)\right\}
$$


If $\operatorname{reg}_{A}\left(X^{\bullet}\right) \neq \operatorname{reg}_{A}\left(Z^{\bullet}\right)+1$, then equality holds.

(3) If $M \in{ }^{*} \bmod A$ has finite length, then $\operatorname{reg}_{A}(M) \leq \max \left\{i \mid M_{i} \neq 0\right\}$.

(4) For $X^{\bullet} \in \mathscr{D}^{\uparrow}(\mathrm{qf} A$ ), we have

$$
\operatorname{reg}_{A}\left(X^{\bullet}\right) \leq \sup \left\{\operatorname{reg}_{A}\left(H^{i}\left(X^{\bullet}\right)\right)+i \mid i \in \mathrm{Z}\right\} .
$$

Proof. (1) is clear. Let us prove (2). Since the triangle yields the long exact sequence $\cdots \rightarrow \underline{\operatorname{Ext}}_{A}^{i}\left(Z^{\bullet}, K\right) \rightarrow \underline{\operatorname{Ext}}_{A}^{i}\left(Y^{\bullet}, K\right) \rightarrow \underline{\operatorname{Ext}}_{A}^{i}\left(X^{\bullet}, K\right) \rightarrow$ $\underline{\operatorname{Ext}}_{A}^{i+1}\left(Z^{\bullet}, K\right) \rightarrow \cdots$, we have the assertions.

We can prove (3) by induction on $\operatorname{dim}_{K} M$. More precisely, if we set $d:=$ $\max \left\{i \mid M_{i} \neq 0\right\}$, we have a short exact sequence $0 \rightarrow K(-d) \rightarrow M \rightarrow$ $M^{\prime} \rightarrow 0$. Now use the induction hypothesis and (2) of this lemma.

In [19, Lemma 2.10], (4) is proved using the spectral sequence

$$
E_{2}^{p, q}=\underline{\operatorname{Ext}}_{A}^{p}\left(H^{-q}\left(X^{\bullet}\right), K\right) \Longrightarrow \underline{\operatorname{Ext}}_{A}^{p+q}\left(X^{\bullet}, K\right)
$$

under the additional assumption that $A$ is regular, left noetherian, and $X^{\bullet}$ is bounded. But these assumptions are clearly irrelevant.

The next result directly follows from Lemma 2.3.

Theorem 3.3 (Eisenbud et al [7], Mori [14]). For $X^{\bullet} \in D^{\uparrow}$ (qf $A$ ), we have

$$
\operatorname{reg}_{A}\left(X^{\bullet}\right)=-\inf \left\{i \mid H^{i}\left(\mathscr{F}_{A}\left(X^{\bullet}\right)\right) \neq 0\right\} .
$$

We say a complex $X^{\bullet} \in \mathscr{D}^{\uparrow}$ (qf $A$ ) is strongly bounded, if $X^{\bullet}$ is bounded (i.e., $H^{i}\left(X^{\bullet}\right)=0$ for $i \gg 0$ and for $i \ll 0$ ) and $\operatorname{reg}_{A}\left(X^{\bullet}\right)<\infty$. Let $\mathscr{D}^{\text {sb }}$ (qf $A$ ) be the full subcategory of $\mathscr{D}^{\uparrow}$ (qf $A$ ) consisting of strongly bounded complexes.

Proposition 3.4. $\mathscr{D}^{\mathrm{sb}}$ (qf $A$ ) is a triangulated subcategory of $\mathscr{D}$ (qf $A$ ).

Proof. Easily follows from Lemma 3.2 (2).

Proposition 3.5. The (restriction of) functors $\mathscr{F}_{A}$ and $\mathscr{F}_{A}$ ! give an equivalence

$$
\mathscr{D}^{\mathrm{sb}}(\mathrm{qf} A) \cong \mathscr{D}^{\mathrm{sb}}\left(\mathrm{qf} A^{!}\right)^{\mathrm{op}} .
$$

Proof. By Theorem 2.2, it suffices to show that $\mathscr{F}_{A}\left(X^{\bullet}\right) \in \mathscr{D}^{\text {sb }}$ (qf $A^{!}$) for all $X^{\bullet} \in \mathscr{D}^{\mathrm{sb}}(\mathrm{qf} A)$. Since $\operatorname{reg}_{A}\left(X^{\bullet}\right)<\infty, \mathscr{F}_{A}\left(X^{\bullet}\right)$ is bounded by Theorem 3.3. Similarly, since $\mathscr{F}_{A^{!}}\left(\mathscr{F}_{A}\left(X^{\bullet}\right)\right)$ is isomorphic to $X^{\bullet}$, which is bounded, we have $\operatorname{reg}_{A^{!}}\left(\mathscr{F}_{A}\left(X^{\bullet}\right)\right)<\infty$.

Let $\left(P^{\bullet}, \partial\right) \in \mathscr{C}^{\uparrow}$ (qf $A$ ) be a complex of free $A$-modules such that $\partial\left(P^{i}\right) \subset$ $\mathrm{m} P^{i+1}$, in other words, $P^{\bullet}$ is a minimal free resolution of some $X^{\bullet} \in \mathscr{C}^{\uparrow}$ (qf $A$ ). According to [7], we define the linear part $\operatorname{lin}\left(P^{\bullet}\right)$ of $P^{\bullet}$ as follows: 
(1) $\operatorname{lin}\left(P^{\bullet}\right)$ is a complex with $\operatorname{lin}\left(P^{\bullet}\right)^{i}=P^{i}$.

(2) The matrices representing the differentials of $\operatorname{lin}\left(P^{\bullet}\right)$ are given by "erasing" all the entries of degree $\geq 2$ (i.e., replacing them by 0 ) from the matrices representing the differentials of $P^{\bullet}$.

It is easy to check that $\operatorname{lin}\left(P^{\bullet}\right)$ is actually a complex. But, even if $P_{\bullet}$ is a minimal free resolution of $M \in$ qf $A, \operatorname{lin}\left(P_{\bullet}\right)$ is not acyclic (i.e., $H_{i}\left(\operatorname{lin}\left(P_{\bullet}\right)\right) \neq 0$ for some $i>0$ ) in general.

Definition 3.6 (Herzog-Iyengar [11]). Let $M \in$ qf $A$ and $P_{\bullet}$ its minimal graded free resolution. We call

$$
\operatorname{ld}_{A}(M):=\sup \left\{i \mid H_{i}\left(\operatorname{lin}\left(P_{\bullet}\right)\right) \neq 0\right\}
$$

the linearity defect of $M$.

We say $M \in{ }^{*} \bmod A$ has a linear (free) resolution if there is some $l \in \mathrm{Z}$ such that $\beta_{i, j}(M) \neq 0$ implies that $j-i=l$. In this case, the minimal free resolution $P_{\bullet}$ of $M$ coincides with $\operatorname{lin}\left(P_{\bullet}\right)$, and $\operatorname{ld}_{A}(M)=0$. For $M \in$ qf $A$ with $\iota:=\inf \left\{i \mid M_{i} \neq 0\right\}, M$ has a linear resolution, if and only if $\operatorname{reg}_{A}(M)=$ $\iota$, if and only if $\operatorname{reg}_{A}(M) \leq \iota$. As shown in [14, Theorem 5.4], we have

$$
\operatorname{reg}_{A}(M)=\inf \left\{i \mid M_{i} \neq 0 \text { and } M_{\geq i}:=\bigoplus_{j \geq i} M_{j} \text { has a linear resolution }\right\} .
$$

For $i \in \mathrm{Z}$ and $M \in \mathrm{qf} A, M_{\langle i\rangle}$ denotes the submodule of $M$ generated by the degree $i$ component $M_{i}$. We say $M \in \mathrm{qf} A$ is componentwise linear, if $M_{\langle i\rangle}$ has a linear resolution for all $i \in \mathrm{Z}$. For example, if $M$ has a linear resolution, then it is componentwise linear. To see this, it suffices to show that if $M=\bigoplus_{i \geq 0} M_{i}$ with $M_{0} \neq 0$ has a linear resolution, then so does $M_{\langle 1\rangle}$. But this follows from the short exact sequence $0 \rightarrow M_{\langle 1\rangle} \rightarrow M \rightarrow M / M_{\langle 1\rangle} \rightarrow 0$ and Lemma 3.2 (2), since $\operatorname{reg}_{A}\left(M / M_{\langle 1\rangle}\right)=\operatorname{reg}_{A}\left(K^{\oplus \operatorname{dim}_{K} M_{0}}\right)=0=\operatorname{reg}_{A}(M)$. Note that $M$ can be componentwise linear even if it is not finitely generated. For example, $\bigoplus_{i \in \mathrm{N}} K(-i)$ is componentwise linear.

Proposition 3.7 (cf. [16], [19]). For $M \in$ qf $A$, the following are equivalent.

(1) $M$ is componentwise linear.

(2) $\operatorname{ld}_{A}(M)=0$.

This result has been proved by Römer [16] and the author [19, Proposition 4.1] under the assumption that $M$ is finitely generated. But this assumption is not important, since for each $j$ the submodule of $M$ generated by $\left\{M_{i} \mid i \leq j\right\}$ is finitely generated. In the proof of [19, Proposition 4.1], the author carelessly stated that "if $M \in{ }^{*} \bmod A$ has a finite length, then 
$\operatorname{reg}_{A}(M)=\max \left\{i \mid M_{i} \neq 0\right\}$ ", which is clearly false (e.g., the exterior algebra $E=\bigwedge\left\langle y_{1}, \ldots, y_{n}\right\rangle$ satisfies $\operatorname{reg}_{E}(E)=0$ while $\left.E_{n} \neq 0\right)$. But the correct statement (Lemma 3.2 (3)) is enough for the proof.

The next result follows easily from Proposition 3.7.

Proposition 3.8 (cf. [16], [19]). For $M \in$ qf $A$, we have

$$
\operatorname{ld}_{A}(M)=\inf \left\{i \mid \Omega_{i}(M) \text { is componentwise linear }\right\},
$$

where $\Omega_{i}(M)$ is the $i^{\text {th }}$ syzygy of $M$.

Clearly, we have $\operatorname{ld}_{A}(M) \leq$ proj. $\operatorname{dim}_{A}(M)$. The inequality is strict quite often. For example, we have proj.dim $\operatorname{dim}_{A}(M)=\infty$ and $\operatorname{ld}_{A}(M)<\infty$ for many $M$. On the other hand, sometimes $\operatorname{ld}_{A}(M)=\infty$.

The next result connects the linearity defect with the regularity via Koszul duality. For a complex $X^{\bullet}, \mathscr{H}\left(X^{\bullet}\right)$ denotes the complex such that $\mathscr{H}\left(X^{\bullet}\right)^{i}=$ $H^{i}\left(X^{\bullet}\right)$ for all $i$ and all differentials are 0 .

Theorem 3.9 (cf. [7, Corollary 3.6]). Let $X^{\bullet} \in \mathscr{D}^{\uparrow}$ (qf $A$ ), and $P^{\bullet} a$ minimal free resolution of $\mathscr{F}_{A}\left(X^{\bullet}\right) \in \mathscr{D}^{\uparrow}\left(\mathrm{qf} A^{!}\right)$. Then we have

$$
\operatorname{lin}\left(P^{\bullet}\right)=F_{A} \circ \mathscr{H}\left(X^{\bullet}\right) .
$$

Hence, for $M \in$ qf $A$,

$$
\operatorname{ld}_{A}(M)=\sup \left\{\operatorname{reg}_{A^{!}}\left(H^{i}\left(F_{A}(M)\right)\right)+i \mid i \in \mathrm{Z}\right\} .
$$

Proof. Recall that the Koszul duality functors used in [7] are covariant, and the $K$-dual of our $\mathscr{F}$. The " $K$-dual version" of the first assertion has been proved in [7, Corollary 3.6] under the assumption that $A$ is the polynomial ring $S$ or the exterior algebra $E$, but the assumption is irrelevant and the proof in [7] also works here. The second assertion follows from Theorem 3.3, since $F_{A}$ ! $\mathscr{H} \circ F_{A}(M)$ is the linear part of the minimal free resolution of $M$.

\section{Koszul Commutative Algebras and their Dual}

If $A$ is a Koszul commutative algebra and $S:=\operatorname{Sym}_{K} A_{1}$ is the polynomial ring, then we have $A=S / I$ for a graded ideal $I$ of $S$. In this situation, $A$ is Golod if and only if $I$ has a 2-linear resolution as an $S$-module (i.e., $\beta_{i, j}(I) \neq 0$ implies $\left.j=i+2\right)$, see [11, Proposition 5.8]. We say A comes from a complete intersection by a Golod map (see [2], [11], although they do not use this terminology), if there is an intermediate graded ring $R$ with $S \rightarrow R \rightarrow A$ satisfying the following conditions:

(1) $R$ is a complete intersection. 
(2) Let $J$ be the graded ideal of $R$ such that $A=R / J$. Then $J$ has a 2-linear resolution as an $R$-module.

If this is the case, $R$ is automatically Koszul (since so is $A$ ). Clearly, if $A$ itself is complete intersection or Golod, then it comes from a complete intersection by a Golod map.

Example 4.1. Set $S=K[s, t, u, v, w]$ and $A=S /(s t, u v, s w)$. Then $A$ is neither Golod nor complete intersection, but comes from a complete intersection by a Golod map (as an intermediate ring, take $S /(s t, u v)$ ).

The next result plays a key role in this section.

Theorem 4.2 (Avramov-Eisenbud [1]). Let A be a Koszul commutative algebra, and $S:=\operatorname{Sym}_{K} A_{1}$ the polynomial ring. Then we have $\operatorname{reg}_{A}(M) \leq$ $\operatorname{reg}_{S}(M)<\infty$ for all $M \in{ }^{*} \bmod A$.

On the other hand, even if $A$ is Koszul and commutative, $\operatorname{ld}_{A}(M)$ can be infinite for some $M \in{ }^{*} \bmod A$, as pointed out in [11]. In fact, if $\operatorname{ld}_{A}(M)<\infty$ then the Poincaré series $P_{M}(t)=\sum_{i \in \mathrm{N}} \beta_{i}(M) \cdot t^{i}$ is rational. But a Koszul commutative algebra $A=K\left[x_{1}, x_{2}, x_{3}\right] /\left(x_{1}, x_{2}, x_{3}\right)^{2} \otimes K\left[x_{4}, x_{5}, x_{6}\right] /\left(x_{4}, x_{5}, x_{6}\right)^{2}$ has a module $M \in{ }^{*} \bmod A$ such that $P_{M}(t)$ is not rational (cf. [17]).

But we have the following.

Theorem 4.3 (Herzog-Iyengar [11]). Let A be a Koszul commutative algebra. If A comes from a complete intersection by a Golod map (e.g., A itself is complete intersection or Golod), then $\operatorname{ld}_{A}(M)<\infty$ for all $M \in{ }^{*} \bmod A$.

Now we are interested in $\operatorname{reg}_{A^{!}}(N)$ and $\operatorname{ld}_{A^{!}}(N)$ for a Koszul commutative algebra $A$. First, we remark the important fact that the categories * mod $A^{!}$and ${ }^{*} \bmod \left(A^{!}\right)^{\text {op }}$ are equivalent in this case. In fact, a graded left $A^{!}$-module has a natural graded right $A^{!}$-module structure, and vice versa (cf. [11, §3]). In particular, $A^{!}$is left noetherian if and only if it is right noetherian.

For the next result and its proof, we need a few preparations. For a graded ring $B=\bigoplus_{i \in \mathrm{N}} B_{i}$, let ${ }^{*} \mathrm{fp} B$ be the full subcategory of * $\bmod B$ consisting of finitely presented modules. We say $B$ is left graded coherent, if any finitely generated graded left ideal of $B$ has a finite presentation. As is well-known, $B$ is left graded coherent if and only if * $\mathrm{fp} B$ is an abelian subcategory of * $\bmod B$.

THEOREM 4.4. If A is a Koszul commutative algebra, we have the following.

(1) Let $N \in{ }^{*} \bmod A^{!}$. If $\operatorname{reg}_{A^{!}}(N)<\infty$, then $\operatorname{ld}_{A^{!}}(N)<\infty$.

(2) The following conditions are equivalent.

(a) $\operatorname{ld}_{A}(M)<\infty$ for all $M \in{ }^{*} \bmod A$.

(a') $\operatorname{ld}_{A}(M)<\infty$ for all $M \in{ }^{*} \bmod A$ with $M=\bigoplus_{i=0,1} M_{i}$.

(b) $\operatorname{reg}_{A^{!}}(N)<\infty$ for all $N \in{ }^{*}$ fp $A^{!}$. 
(3) Let $N \in$ qf $A$ !. If there is some $c \in \mathrm{N}$ such that $\operatorname{dim}_{K} N_{i} \leq c$ for all $i \in \mathrm{Z}$, then $\operatorname{ld}_{A^{!}}(N)<\infty$.

Proof. (1) The complex $F_{A^{!}}(N)$ is always bounded above. Hence if $\operatorname{reg}_{A^{!}}(N)<\infty$ then $H^{i}\left(F_{A^{!}}(N)\right) \neq 0$ for only finitely many $i$ by Theorem 3.3. Thus the assertion follows from Theorems 3.9 and 4.2.

(2) The implication (a) $\Rightarrow\left(a^{\prime}\right)$ is clear.

$\left(\mathrm{a}^{\prime}\right) \Rightarrow$ (b): First assume that $N \in{ }^{*} \mathrm{fp} A^{!}$has a presentation of the form $A^{!}(-1)^{\oplus \beta_{1}} \rightarrow A^{! \oplus \beta_{0}} \rightarrow N \rightarrow 0$. Then there is $M \in{ }^{*} \bmod A$ with $M=$ $\bigoplus_{i=0,1} M_{i}$ such that $F_{A}(M)$ gives this presentation. Since $\operatorname{ld}_{A}(M)<\infty$, we have $\operatorname{reg}_{A^{!}}(N)<\infty$ by Theorem 3.9.

Next take an arbitrary $N \in{ }^{*}$ fp $A$ !. For a sufficiently large $s, N_{\geq s}:=$ $\bigoplus_{i \geq s} N_{i}$ has a presentation of the form $A^{!}(-s-1)^{\oplus \beta_{1}} \rightarrow A^{!}(-s)^{\oplus \beta_{0}} \rightarrow$ $N_{\geq s} \rightarrow 0$. (To see this, consider the short exact sequence $0 \rightarrow N_{\geq s} \rightarrow N \rightarrow$ $N / N_{\geq s} \rightarrow 0$, and use the fact that $\operatorname{reg}_{A^{!}}\left(N / N_{\geq s}\right)<s$. We have shown that $\operatorname{reg}_{A^{!}}\left(N_{\geq s}\right)<\infty$. So reg $A^{!}(N)<\infty$ by the above short exact sequence.

(b) $\Rightarrow$ (a): First, we show that $A^{!}$is left graded coherent in this case. Assume the contrary. Then there is a finitely generated graded left ideal $I \subset A^{!}$which is not finitely presented. Clearly, $A^{!} / I$ has a finite presentation, but $\beta_{2}\left(A^{!} / I\right)=$ $\beta_{1}(I)=\infty$, in particular, $\operatorname{reg}_{A^{!}}\left(A^{!} / I\right)=\infty$. This is a contradiction.

So ${ }^{*} \mathrm{fp} A^{!}$is an abelian category. Each term of $F_{A}(M)$ is a finite free $A^{!}$module, in particular, $F_{A}(M) \in \mathscr{C}^{-}\left({ }^{*} \mathrm{fp} A^{!}\right)$. Hence we have $H^{i}\left(F_{A}(M)\right) \in$ ${ }^{*} \mathrm{fp} A^{!}$for all $i$. By the assumption, $\operatorname{reg}_{A^{!}}\left(H^{i}\left(F_{A}(M)\right)\right)<\infty$. On the other hand, $H^{i}\left(F_{A}(M)\right) \neq 0$ for finitely many $i$ by Theorems 3.3 and 4.2. So the assertion follows from Theorem 3.9.

(3) Let $\mathscr{S}$ be the set of all graded submodules of $A^{\oplus c}$ which are generated by elements of degree 1. By Brodmann [5], there is some $C \in \mathrm{N}$ such that $\operatorname{reg}_{A}(M) \leq \operatorname{reg}_{S}(M)<C$ for all $M \in \mathscr{S}$. Here $S$ denotes the polynomial ring $\operatorname{Sym}_{K} A_{1}$. To prove the assertion, it suffices to show that $\operatorname{reg}_{A}\left(H^{i}\left(\mathscr{F}_{A}(N)\right)\right)+$ $i<C$ for all $i$. We may assume that $i=0$. Note that $H^{0}\left(\mathscr{F}_{A} !(N)\right)$ is the cohomology of the sequence

$$
A \otimes_{K}\left(N_{1}\right)^{*} \stackrel{\partial^{-1}}{\longrightarrow} A \otimes_{K}\left(N_{0}\right)^{*} \stackrel{\partial^{0}}{\longrightarrow} A \otimes_{K}\left(N_{-1}\right)^{*} .
$$

Since $\operatorname{Im}\left(\partial^{0}\right)(-1)$ is a submodule of $A^{\oplus \operatorname{dim}_{K} N_{-1}}$ generated by elements of degree 1 and $\operatorname{dim}_{K} N_{-1} \leq c$, we have $\operatorname{reg}_{A}\left(\operatorname{Im}\left(\partial^{0}\right)\right)<C-1$. Consider the short exact sequence

$$
0 \longrightarrow \operatorname{Ker}\left(\partial^{0}\right) \longrightarrow A \otimes_{K}\left(N_{0}\right)^{*} \longrightarrow \operatorname{Im}\left(\partial^{0}\right) \longrightarrow 0 .
$$

Since $\operatorname{reg}_{A}\left(A \otimes_{K}\left(N_{0}\right)^{*}\right)=0$, we have $\operatorname{reg}_{A}\left(\operatorname{Ker}\left(\partial^{0}\right)\right)<C$. Similarly, we 
have $\operatorname{reg}_{A}\left(\operatorname{Im}\left(\partial^{-1}\right)\right)<C$. By the short exact sequence

$$
0 \longrightarrow \operatorname{Im}\left(\partial^{-1}\right) \longrightarrow \operatorname{Ker}\left(\partial^{0}\right) \longrightarrow H^{0}\left(\mathscr{F}_{A^{!}}(N)\right) \longrightarrow 0,
$$

we are done.

Remark 4.5. In Theorem 4.4 (2), the implications (a) $\Rightarrow\left(a^{\prime}\right) \Leftrightarrow$ (b) hold for a general Koszul algebra.

If $A$ is a (not necessarily commutative) Koszul algebra satisfying $\operatorname{reg}_{A}(M)<\infty$ for all $M \in{ }^{*} \bmod A$, then Theorem 4.4 (1) and (2) hold for $A$.

In [2, Corollary 3], Backelin and Roos showed that if $A$ is a Koszul commutative algebra which comes from a complete intersection by a Golod map then $A^{!}$is left graded coherent. Moreover, they actually proved that $\operatorname{reg}_{A^{!}}(N)<\infty$ for all $N \in{ }^{*} \mathrm{fp} A^{!}$(see [2, Corollary 2] and [11, Lemma 5.1]). So we have $\operatorname{ld}_{A}(M)<\infty$ for all $M \in{ }^{*} \bmod A$ by Theorem 4.4, that is, we get a result of Herzog and Iyengar (Theorem 4.3). Their original proof is essentially based on this line too.

A deep theory on the Hopf algebra structure of $A^{!}$plays a key role in [2]. But, when $A$ is a Koszul complete intersection, we have another exposition of the fact that $\operatorname{reg}_{A^{!}}(N)<\infty$ for all $N \in{ }^{*} \bmod A^{!}$. Since this exposition has its own interest, we will give it here. The next lemma might be known to specialists. But the author could not find a reference. So we give a proof, which is suggested by Professor Izuru Mori. For the unexplained terminology appearing in the next result and its proof, consult [14], [18], [20].

Lemma 4.6. If $A$ is a complete intersection, then $A^{!}$is noetherian and admits a balanced dualizing complex.

Proof. Let $S:=\operatorname{Sym}_{K} A_{1}$ be the polynomial ring. We have a regular sequence $z_{1}, \ldots, z_{m} \in S_{2}$ such that $A=S /\left(z_{1}, \ldots, z_{m}\right)$. Recall that $E:=S^{!}$is the exterior algebra. Set $A_{(1)}:=S /\left(z_{1}\right)$. Then there is a central regular element $w_{1} \in\left(A_{(1)}\right)^{!}$of degree 2 such that $\left(A_{(1)}\right)^{!} /\left(w_{1}\right) \cong E$ by [18, Theorem 5.12]. Since $E$ is artinian, it is noetherian and admits a balanced dualizing complex. Hence $\left(A_{(1)}\right)$ ! is noetherian and admits a balanced dualizing complex too by [14, Lemma 7.2]. Similarly, if we set $A_{(2)}:=S /\left(z_{1}, z_{2}\right)=A_{(1)} /\left(\bar{z}_{2}\right)$, then there is a central regular element $w_{2} \in\left(A_{(2)}\right)$ ! of degree 2 such that $\left(A_{(2)}\right)^{!} /\left(w_{2}\right) \cong\left(A_{(1)}\right)^{!}$. Hence $\left(A_{(2)}\right) !$ is noetherian and admits a balanced dualizing complex again. Repeating this argument, we see that $A$ ! is noetherian and has a balanced dualizing complex.

Corollary 4.7. If $A$ is a Koszul complete intersection, then $\operatorname{reg}_{A^{!}}(N)<\infty$ and $\operatorname{ld}_{A^{!}}(N)<\infty$ for all $N \in{ }^{*} \bmod A^{!}$. 
Proof. By Lemma 4.6 and [12], we have $\operatorname{reg}_{A^{!}}(N)<\infty$ for all $N \in$ ${ }^{*} \bmod A^{!}$. Hence $\operatorname{ld}_{A^{!}}(N)<\infty$ for all $N \in{ }^{*} \bmod A^{!}$by Theorem 4.4.

The next result, which easily follows from [11, Corollary 6.2], states that if $A$ is Golod then there is an upper bound of $\operatorname{reg}_{A^{!}}(N)$ for $N \in{ }^{*} \mathrm{fp} A^{!}$depending only on $\max \left\{j \mid \beta_{1, j}(N) \neq 0\right\}$. Even if $A=K\left[x_{1}, \ldots, x_{n}\right], n \geq 2$, there is no similar bound for $\operatorname{reg}_{A}(M)$ at all.

Proposition 4.8. Assume that $A$ is Koszul and Golod. For $N \in{ }^{*} \mathrm{fp} A !$, we have

$$
\operatorname{reg}_{A^{!}}(N)<2 \cdot \operatorname{dim}_{K} A_{1}+\max \left\{j \mid \beta_{1, j}(N) \neq 0\right\} .
$$

Proof. Assume that $N \in{ }^{*} \mathrm{fp} A^{!}$has a presentation of the form $A^{!}(-1)^{\oplus \beta_{1}} \rightarrow$ $A^{! \oplus \beta_{0}} \rightarrow N \rightarrow 0$. Then there is $M \in{ }^{*} \bmod A$ with $M=\bigoplus_{i=0,1} M_{i}$ such that $F_{A}(M)$ gives this presentation. Since $\operatorname{ld}_{A}(M) \leq 2 \cdot \operatorname{dim}_{K} A_{1}$ by [11, Corollary 6.2], we have $\operatorname{reg}_{A^{!}}(N) \leq 2 \cdot \operatorname{dim}_{K} A_{1}$ by Theorem 3.9.

Next take an arbitrary $N \in{ }^{*} \mathrm{fp} A^{!}$and set $s:=\max \left\{j \mid \beta_{1, j}(N) \neq\right.$ $0\}$. Then $N^{\prime}:=\bigoplus_{i \geq s-1} N_{i}$ has a presentation of the form $A^{!}(-s)^{\oplus \beta_{1}} \rightarrow$ $A^{!}(-s+1)^{\oplus \beta_{0}} \rightarrow N^{\prime} \rightarrow 0$, and we have $\operatorname{reg}_{A^{!}}(N)=\operatorname{reg}_{A^{!}}\left(N^{\prime}\right)$. Since $N^{\prime}(s-1)$ satisfies the conditon in the previous paragraph, we have $\operatorname{reg}_{A^{!}}(N)=$ $\operatorname{reg}_{A^{!}}\left(N^{\prime}\right)<2 \operatorname{dim}_{K} A_{1}+s$.

Even if $A$ is not commutative, $\operatorname{ld}_{A}(M)<\infty$ for all $M \in{ }^{*} \bmod A$ implies that $\operatorname{reg}_{A^{!}}(N)<\infty$ for all $N \in{ }^{*} \mathrm{fp} A^{!}$as noted in Remark 4.5. So we have the following (see the proof of the implication (b) $\Rightarrow$ (a) of Theorem 4.4 (2)).

Proposition 4.9. Let $A$ be a Koszul algebra. If $\operatorname{ld}_{A}(M)<\infty$ for all $M \in{ }^{*} \bmod A$, then $A^{!}$is left graded coherent.

Lemma 4.10. Let $A$ be a Koszul algebra with $\operatorname{ld}_{A}(M)<\infty$ for all $M \in$ ${ }^{*} \bmod A$, and let $X^{\bullet} \in \mathscr{D}^{b}\left(\mathrm{qf} A^{!}\right)$be a bounded complex. Then $\operatorname{reg}_{A^{!}}\left(X^{\bullet}\right)<\infty$ if and only if $H^{i}\left(X^{\bullet}\right) \in{ }^{*} \mathrm{fp} A^{!}$for all $i$.

Proof. (Sufficiency): If $H^{i}\left(X^{\bullet}\right) \in{ }^{*} \mathrm{fp} A^{!}$, then $\operatorname{reg}_{A^{!}}\left(H^{i}\left(X^{\bullet}\right)\right)<\infty$. Since $X^{\bullet}$ is bounded, we have $\operatorname{reg}_{A^{!}}\left(X^{\bullet}\right)<\infty$ by Lemma 3.2 (4).

(Necessity): Assume that $\operatorname{reg}_{A^{!}}\left(X^{\bullet}\right)<\infty$ (more generally, $\beta^{i}\left(X^{\bullet}\right)<\infty$ for all $i$ ), and $P^{\bullet}$ is a minimal free resolution of $X^{\bullet}$. Clearly, $P^{i} \in{ }^{*} \mathrm{fp} A^{!}$for each $i$. By Proposition 4.9, ${ }^{*} \mathrm{fp} A^{!}$is an abelian category and each $H^{i}\left(P^{\bullet}\right)\left(\cong H^{i}\left(X^{\bullet}\right)\right)$ belongs to *fp $A$ !.

If $A$ is commutative, then $A$ is noetherian and ${ }^{*} \bmod A$ is an abelian category. So we can consider the derived category $\mathscr{D}^{b}\left({ }^{*} \bmod A\right)$, which is a full subcategory of $\mathscr{D}^{\uparrow}$ (qf $\left.A\right)$. 
Lemma 4.11. Let $A$ be a Koszul commutative algebra. Then $\mathscr{D}^{b}\left({ }^{*} \bmod A\right)=$ $\mathscr{D}^{\mathrm{sb}}(\mathrm{qf} A)$ and the Koszul duality gives $\mathscr{D}^{b}\left({ }^{*} \bmod A\right) \cong \mathscr{D}^{\mathrm{sb}}\left(\mathrm{qf} A^{!}\right)^{\mathrm{op}}$.

Proof. By Proposition 3.5, it suffices to show the first statement. If $X^{\bullet} \in$ $\mathscr{D}^{b}\left({ }^{*} \bmod A\right)$, then $\operatorname{reg}_{A}\left(X^{\bullet}\right)<\infty$ by Lemma 3.2 (4) and Theorem 4.2. Hence we have $X^{\bullet} \in \mathscr{D}^{\mathrm{sb}}(\mathrm{qf} A)$. Conversely, if $Y^{\bullet} \in \mathscr{D}^{\mathrm{sb}}(\mathrm{qf} A)$, then $\beta^{i}\left(Y^{\bullet}\right)<\infty$ for all $i$, and the minimal free resolution of $Y^{\bullet}$ is a complex of finite free modules. So we have $Y^{\bullet} \in \mathscr{D}^{b}\left({ }^{*} \bmod A\right)$.

THEOREM 4.12. Let A be a Koszul commutative algebra such that $\operatorname{ld}_{A}(M)<$ $\infty$ for all $M \in{ }^{*} \bmod A$ (e.g., A comes from a complete intersection by a Golod map). Then Koszul duality gives an equivalence $\mathscr{D}^{b}\left({ }^{*} \bmod A\right) \cong \mathscr{D}^{b}\left({ }^{*} \mathrm{fp} A^{!}\right)^{\mathrm{op}}$.

Proof. By Proposition 4.9, ${ }^{*} \mathrm{fp} A$ ! is an abelian category, and closed under extensions in qf $A^{!}$. Since a free $A^{!}$-module of finite rank belongs to *fp $A^{!}$, this category has enough projectives. So we have $\mathscr{D}^{b}\left({ }^{*}\right.$ fp $\left.A^{!}\right)=\mathscr{D}_{* \text { fp } A^{!}}^{b}\left(\right.$ qf $\left.A^{!}\right)=$ $\mathscr{D}^{\text {sb }}$ (qf $A^{!}$). Here the first equality follows from [9, Exercise III.2.2] and the second one follows from Lemma 4.10. Now the assertion is a direct consequence of Lemma 4.11.

We remark that the next corollary also follows from Lemma 4.6 and [14, Proposition 4.5].

COROLlaRY 4.13. If A is a Koszul complete intersection, then Koszul duality gives $\mathscr{D}^{b}\left({ }^{*} \bmod A\right) \cong \mathscr{D}^{b}\left({ }^{*} \bmod A^{!}\right)^{\text {op }}$.

In the rest of the paper, we study the linearity defect over the exterior algebra $E:=\bigwedge\left\langle y_{1}, \ldots, y_{n}\right\rangle$. Eisenbud et al. [7] showed that $\operatorname{ld}_{E}(N)<\infty$ for all $N \in{ }^{*} \bmod E$. Now this is a special case of Theorem 4.4. But the behavior of $\operatorname{ld}_{E}(N)$ is still mysterious.

If $n \geq 2$, then we have $\sup \left\{\operatorname{ld}_{E}(N) \mid N \in{ }^{*} \bmod E\right\}=\infty$. In fact, $N:=$ $E / \operatorname{soc}(E)$ satisfies $\operatorname{ld}_{E}(N) \geq 1$. And the $i^{\text {th }} \operatorname{cosyzygy} \Omega_{-i}(N)$ of $N$ (since $E$ is selfinjective, we can consider cosyzygies) satisfies $\operatorname{ld}_{E}\left(\Omega_{-i}(N)\right)>i$. But we have an upper bound of $\operatorname{ld}_{E}(N)$ depending only on $\max \left\{\operatorname{dim}_{K} N_{i} \mid i \in \mathrm{Z}\right\}$ and $n$. Before stating this, we recall a result on $\operatorname{reg}_{S}(M)$ for $M \in{ }^{*} \bmod S$.

Theorem 4.14 (Brodmann and Lashgari, [6, Theorem 2.6]). Let $S=$ $k\left[x_{1}, \ldots, x_{n}\right]$ be the polynomial ring. Assume that a graded submodule $M \subset$ $S^{\oplus c}$ is generated by elements whose degrees are at most $d$. Then we have $\operatorname{reg}_{S}(M) \leq c^{n !}(2 d)^{(n-1) !}$.

When $c=1$ (i.e., when $M$ is an ideal), the above bound is a classical result, and there is a well-known example which shows the bound is rather sharp. For our study on $\operatorname{ld}_{E}(N)$, the case when $d=1$ (but $c$ is general) is essential. When 
$c=d=1$, we always have $\operatorname{reg}_{S}(M)=1$. So the author believes the bound can be strongly improved when $d=1$.

Proposition 4.15. Let $E=\bigwedge\left\langle y_{1}, \ldots, y_{n}\right\rangle$ be an exterior algebra, and $N \in{ }^{*} \bmod E$. Set $c:=\max \left\{\operatorname{dim}_{K} N_{i} \mid i \in \mathrm{Z}\right\}$. Then $\operatorname{ld}_{E}(N) \leq c^{n !} 2^{(n-1) !}$.

Proof. If $M$ is a graded submodules of $S^{\oplus c}$ generated by elements of degree 1 , then we have $\operatorname{reg}_{S}(M) \leq c^{n !} 2^{(n-1) !}$ by Theorem 4.14 . Now the assertion follows from the argument similar to the proof of Theorem 4.4 (3).

AcKNOWLEDGMents. The author is grateful to Professors Izuru Mori and Ryota Okazaki for useful comments.

\section{REFERENCES}

1. Avramov, L., and Eisenbud, D., Regularity of modules over a Koszul algebra, J. Algebra 153 (1992), 85-90.

2. Backelin, J., Roos, J.-E., When is the double Yoneda Ext-algebra of a local Noetherian ring again Noetherian?, pp. 101-119 in: Algebra, Algebraic Topology and their Interactions (Stockholm 1983), Lecture Notes in Math. 1183, Springer, Berlin 1986.

3. Beilinson, A., Ginzburg, V., and Soergel, W., Koszul duality patterns in representation theory, J. Amer. Math. Soc. 9 (1996), 473-527.

4. Bøgvad, R., and Halperin, S., On a conjecture of Roos, pp. 120-127 in: Algebra, Algebraic Topology and their Interactions (Stockholm 1983), Lecture Notes in Math. 1183, Springer, Berlin 1986.

5. Brodmann, M., Castelnuovo-Mumford regularity and degrees of generators of graded submodules, Illinois J. Math. 47 (2003), 749-767.

6. Brodmann, M., and Lashgari, A. F., A diagonal bound for cohomological postulation numbers of projective schemes, J. Algebra 265 (2003), 631-650.

7. Eisenbud, D., Fløystad, G., and Schreyer, F.-O., Sheaf cohomology and free resolutions over exterior algebras, Trans. Amer. Math. Soc. 355 (2003), 4397-4426.

8. Eisenbud, D., and Goto, S., Linear free resolutions and minimal multiplicity, J. Algebra 88 (1984), 89-133.

9. Gelfand, S. I., and Manin, Y. I., Methods of Homological Algebra, Springer, Berlin 1996.

10. Herzog, J., and Hibi, T., Componentwise linear ideals, Nagoya Math. J. 153 (1999), 141-153.

11. Herzog, J., and Iyengar, S., Koszul modules, J. Pure Appl. Algebra 201 (2005), 154-188.

12. Jørgensen, P., Linear free resolutions over non-commutative algebras, Compos. Math. 140 (2004), 1053-1058.

13. Martínez-Villa, R., and Zacharia, D., Approximations with modules having linear resolutions, J. Algebra 266 (2003), 671-697.

14. Mori, I., Rationality of the Poincaré series for Koszul algebras, J. Algebra 276 (2004), 602 624.

15. Okazaki, R., and Yanagawa, K., Linearity defects of face rings, J. Algebra 314 (2007), 362382.

16. Römer, T., On minimal graded free resolutions, Thesis, University of Essen, 2001.

17. Roos, J.-E., Good and bad Koszul algebras and their Hochschild homology, J. Pure Appl. Algebra 201 (2005), 295-327. 
18. Smith, S. P., Some finite dimensional algebras related to elliptic curves, pp. 315-348 in: Representation Theory of Algebras and Related Topics (Mexico City 1994), CMS Conference Proceedings 19, Amer. Math. Soc., Providence, RI 1996.

19. Yanagawa, K., Castelnuovo-Mumford regularity for complexes and weakly Koszul modules, J. Pure Appl. Algebra 207 (2006), 77-97.

20. Yekutieli, A., Dualizing complexes over noncommutative graded algebras, J. Algebra 153 (1992), 41-84.

DEPARTMENT OF MATHEMATICS

FACULTY OF ENGINEERING SCIENCE

KANSAI UNIVERSITY

SUITA 564-8680

JAPAN

E-mail: yanagawa@ipcku.kansai-u.ac.jp 